$\operatorname{CoN} f-9511149-1$

UCRL-JC-122277

PREPRINT

\title{
Dynamics of Laser Excitation, Ionization and Harmonic Conversion in Inert Gas Atoms
}

Kenneth C. Kulander

RECFIVED

fillis 1 ; 1995

UST.I

This was prepared for submittal to the Progress in Crystal Growth and Characterizations

of Materials International Symposium

November 8-10, 1995

Tokyo, Japan

October 1995

This is a preprint of a paper intended for publication in a journal or proceedings. Since changes may be made before publication, this preprint is made available with the understanding that it will not be cited or reproduced without the permission of the author.

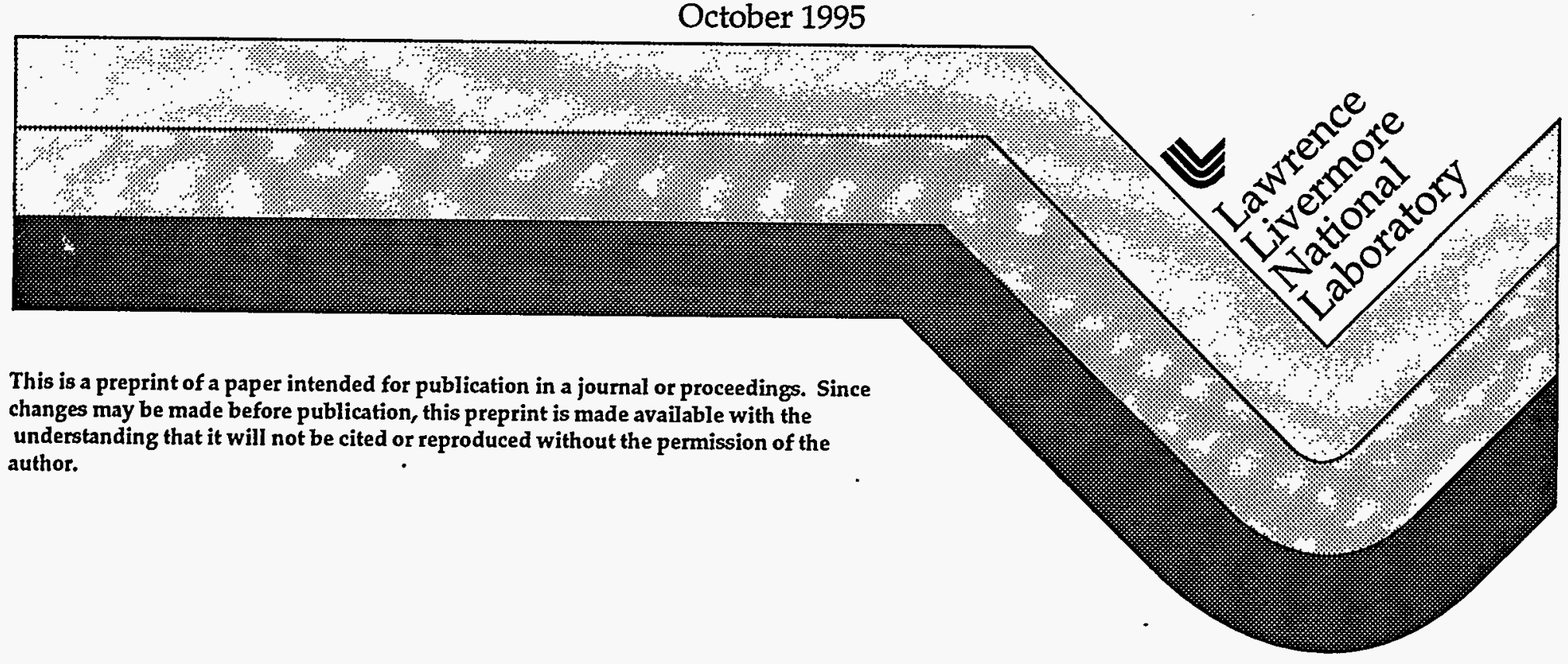




\section{DISCLAIMER}

This document was prepared as an account of work sponsored by an agency of the United States Government. Neither the United States Government nor the University of California nor any of their employees, makes any warranty, express or implied, or assumes any legal liability or responsibility for the accuracy, completeness, or usefulness of any information, apparatus, product, or process disclosed, or represents that its use would not infringe privately owned rights. Reference herein to any specific commercial product, process, or service by trade name, trademark, manufacturer, or otherwise, does not necessarily constitute or imply its endorsement, recommendation, or favoring by the United States Government or the University of California. The views and opinions of authors expressed herein do not necessarily state or reflect those of the United States Government or the University of California, and shall not be used for advertising or product endorsement purposes. 


\title{
DYNAMICS OF LASER EXCITATION, IONIZATION AND HARMONIC CONVERSION IN INERT GAS ATOMS
}

\author{
Kenneth C. Kulander \\ Theoretical Atomic and Molecular Physics Group \\ Physics Directorate \\ Lawrence Livermore National Laboratory, Livermore, CA 94551
}

\begin{abstract}
Studies of non-perturbative multiphoton processes in atoms in pulsed laser fields employ a single-activeelectron (SAE) model which follows the time evolution of each valence electron in the frozen, mean-field of the remaining electrons, the nucleus and the laser field. The photoelectron and photon emission spectra, although related, are not identical. A simple two-step, quasi-classical model explains the differences and gives a more complete understanding of the strong field induced dynamics.
\end{abstract}

\section{INTRODUCTION}

In recent years there have been significant advances in short pulse, high intensity laser technology. Lasers with pulse lengths of $0.02-1$ ps and wavelengths from $0.2-1 \mu \mathrm{m}$ can be focused to produce intensities from $10^{12}$ to above $10^{20}$ $\mathrm{W} / \mathrm{cm}^{2}$ [1]. One use of these systems has been to study the response of atoms to such intense, well characterized electromagnetic fields. Because these pulses are very short, neutral atoms survive to experience intensities where traditional perturbation expansions of the wave function in terms of the field-free states fails completely to describe the dynamics of the system. One approach which can represent these strong field effects employs an explicit, non-perturbative timedependent solution to the Schrödinger equation.

Evidence for strong field non-perturbative dynamics can be found, for example, in the measured photoelectron energy distributions which exhibit a series of peaks separated by the photon energy. These spectra demonstrate a high probability for the absorption of many more photons than the minimum required for ionization. This phenomenon is called above threshold ionization (ATI). A second remarkable high intensity effect is the efficient generation of very high-order harmonic radiation during the laser pulse. In the direction of propagation of the 
incident beam, strong coherent emission at odd multiples of the driving frequency is produced. The even harmonics are forbidden by symmetry. Harmonics up to the 109th order of a $140 \mathrm{fs}$ Ti-sapphire $(806 \mathrm{~nm})$ laser [2], the 133rd harmonic of a 1053 $\mathrm{nm} 1$ ps Nd-YLF laser [3] and the 25th harmonic of a $300 \mathrm{fs} \mathrm{KrF}$ (248 nm) laser [4] have been obtained in neon. There is good evidence in the last case [4] that the highest harmonics are being produced from either the singly or possible the doubly ionized atoms. In general, however, the high order harmonics are generated as the neutral atoms are being ionized.

\section{METHOD}

A many electron atom in an intense, pulsed laser field is a formidable computational problem. This is because the Hamiltonian,

$$
H(t)=H_{o}+V_{I}(t)
$$

is explicitly time dependent. $H_{O}$ is the (non-relativistic) atomic Hamiltonian and $V_{I}(t)$ is the interaction between the electron and the field. The laser is assumed to be linearly polarized along the $z$-axis and the field is strong enough to be treated semiclassically, giving

$$
V_{I}(t)=e \sum_{i} z_{i} \varepsilon_{o} f(t) \sin (\omega t)
$$

Here $\varepsilon_{o}$ is the magnitude of the field and $\omega$ is the frequency. The pulse envelope, $f(t)$, is typically chosen to be either a sine-squared or trapezoidal pulse. If we are interested in calculating quantities relevant to a particular intensity, $I_{O}=c \varepsilon_{o} 2 / 8 \pi$, we choose a pulse envelope that rises over several optical cycles to its maximum value (1.0) and then is held constant for 20-30 cycles.

In many of the short-pulse experiments carried out today, $V_{I}(t)$ is comparable in strength to the Coulombic interactions within the atom. Therefore the method of solution must be able to treat these different interactions on an equal footing. Because the full multi-electron problem is beyond present computational capabilities, we have developed a model based on the Hartree-Fock method in which the time-dependent wave function in approximated by a product of single particle orbitals. This approach can provide considerable insight into the excitation dynamics as well as quantitative predictions for the observed multiphoton processes.

The calculations reported here have been performed using the single-activeelectron (SAE) approximation [5]. In this model we hold the orbitals of all but one of the electrons in the atom fixed and allow the active electron to respond to the 
laser in the mean field of the remaining electrons and the nucleus. The effective potentials for the active electron are generated from field-free Hartree-Slater calculations for the ground and singly-excited states of the atom. Thus we solve the time-dependent Schrödinger equation,

$$
i \hbar \frac{\partial}{\partial t} \psi_{j}(r, t)=H_{j}(t) \psi_{j}(r, t)
$$

for the active-electron orbital, $\psi_{j}(r, t)$, which at $t=0$ is the $j$ th valence pseudo-orbital. $H_{j}$ contains the appropriate effective potentials and the interaction between the laser field and this electron. Ionization and photoemission rates are determined during the last part of the pulse, after the transient excitations have decayed by ionization over a few cycles following the ramp. Our finite difference integration of Eq. (3) is carried out on a grid with absorbing boundaries. The equation for the orbital wave functions has cylindrical symmetry so the spatial representation requires only two dimensions. We obtain ionization rates by monitoring the norm of the wave function. As flux reaches the edges of the integration volume it is removed. The rate at which this occurs is defined to be the ionization rate.

For energy and angular distributions of the ejected electrons, we choose a pulse which also turns off over a few additional cycles (a trapezoidal pulse) and then perform an analysis of the final wave function using an energy window function [6].

The emission strengths at the harmonic frequencies are proportional to the square of the Fourier transform of the total induced dipole, which we typically calculate over the last five cycles of the pulse described above:

$$
d_{q}=\frac{1}{T_{f}-T_{i}} \sum_{j} \int_{T_{i}}^{T_{f}} d t e^{-i q \omega t}\left\langle\psi_{j}(t)|z| \psi_{j}(t)\right\rangle .
$$

In fact we have found it better to use the acceleration form of the dipole, which is numerically more tractable, for determining the time-dependent dipole.

\section{RESULTS}

We have calculated rates for photon and electron emission rates as described above for xenon for intensities in the range from $1-5 \times 10^{13} \mathrm{~W} / \mathrm{cm}^{2}$. The results are shown in Figure 1 where we compare the single-atom emission strengths for the two processes at four different intensities. The harmonic emission strength is given by $\omega^{3}$ times $\left|d_{q}\right|^{2}$. We can see that there is a distinct difference in that there is a dramatic cutoff in the harmonic spectrum that is not present in ATI. However below the harmonic cutoff the harmonic and ATI strengths are clearly very similar. By 
comparing calculated photoemission spectra for many systems we conclude that this harmonic cutoff scales linearly with the laser intensity, and is given approximately by the simple expression $I_{p}+3 U_{p}$, where $I_{p}$ is the ionization potential and $U_{p}=I_{o} /\left(4 \omega^{2}\right)$ is the ponderomotive energy of a free electron in the laser field [7]. We have also noted these predicted cutoffs in figure 1 . These results show that it is possible that there is may not be a simple, direct connection between the ATI and harmonic spectra.

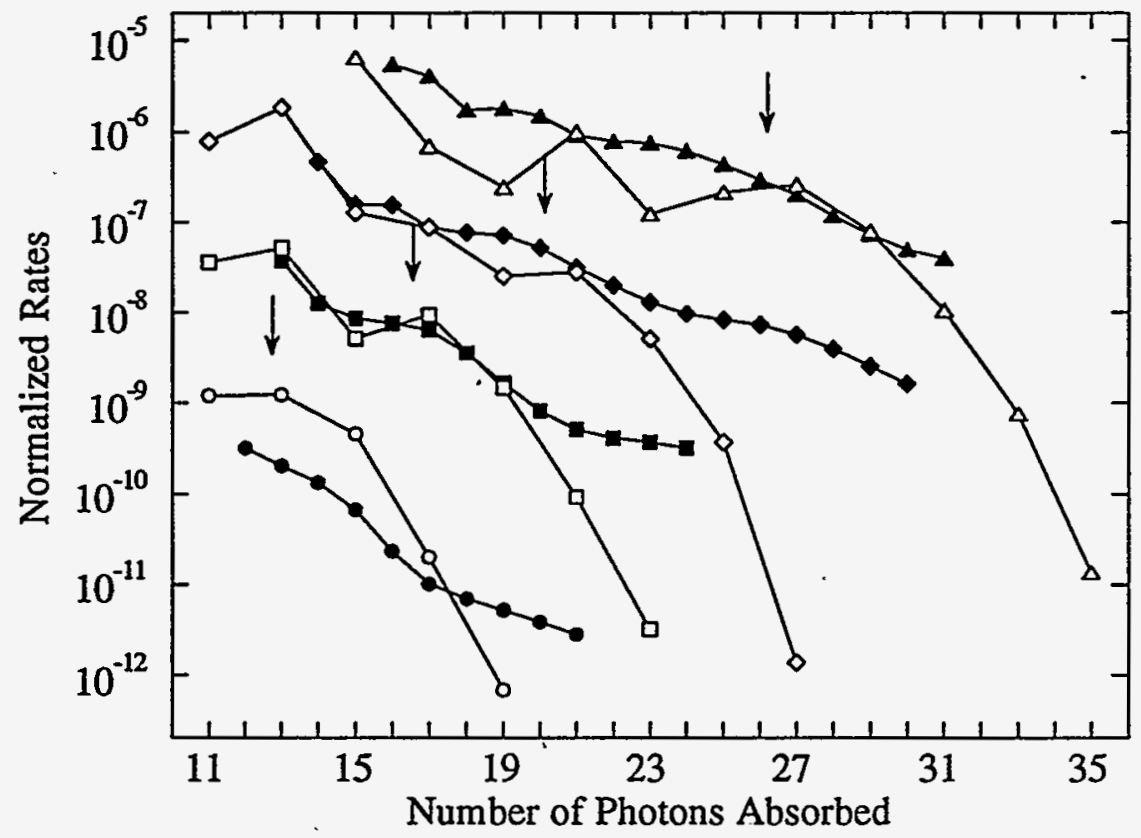

Fig. 1 Photon (open symbols) and electron (filled symbols) emission rates for Xe at $1.064 \mu \mathrm{m}$ and intensities of 1 (circles), 2 (squares), 3 (triangles) and 5 (diamonds) $\times 10^{14} \mathrm{~W} / \mathrm{cm}^{2}$. The harmonic rates are scaled to agree with the electron rate for the 21 st order peaks for the highest intensity shown. The arrows denote the predicted harmonic cutoff at $=I_{p}+3 U_{p}$.

\section{TWO-STEP MODEL}

We can understand this difference by considering a simple quasi-classical model for the ionization process [8-10]. The model assumes multiphoton ionization can be separated into two steps. The first, initiation, step is when the laser field promotes some probability from the ground state into the continuum. Each half optical cycle a continuum wave packet is created. This wave packet evolves primarily in response to the oscillating electromagnetic field because it is mostly well removed from the influence of the ionic core potential. We consider this propagation within the continuum the second step of this model and, since the density of states within the continuum is high, we can approximate the wave packet evolution with a collection of classical trajectories. Thus at each instant of time during the optical cycle we assume a probability for a quantum transition into the continuum of a point particle which then evolves according to the classical 
equations of motion for an electron in the laser field and subject to its interaction with the ion core. For a free electron these are:

$$
\begin{aligned}
& \ddot{z}(t)=-\frac{e \varepsilon_{o}}{m} \sin \left(\omega t+\omega t_{\text {init }}\right) \\
& \dot{z}(t)=\frac{e \varepsilon_{o}}{m \omega} \cos \left(\omega t+\omega t_{\text {init }}\right)-\frac{e \varepsilon_{o}}{m \omega} \cos \left(\omega t_{\text {init }}\right)
\end{aligned}
$$

and

$$
z(t)=\frac{e \varepsilon_{o}}{m \omega^{2}} \sin \left(\dot{\omega} t+\omega t_{i n i t}\right)-\frac{e \varepsilon_{o} t}{m \omega} \cos \left(\omega t_{\text {init }}\right)+\frac{e \varepsilon_{o}}{m \omega^{2}} \sin \left(\omega t_{\text {init }}\right)
$$

where $t_{\text {init }}$ is the time the electron is freed. In the velocity equation (6) the first term in the quiver velocity induced by the oscillating field and the drift velocity is the second term. The drift velocity depends on the phase of the field when the electron enters the continuum.

In figure 2 we show the phase space plot of one such trajectory. The electron starts near the nucleus and is initially pushed away from the ion core during the next quarter cycle. Then the electric field changes direction and pushes the electron

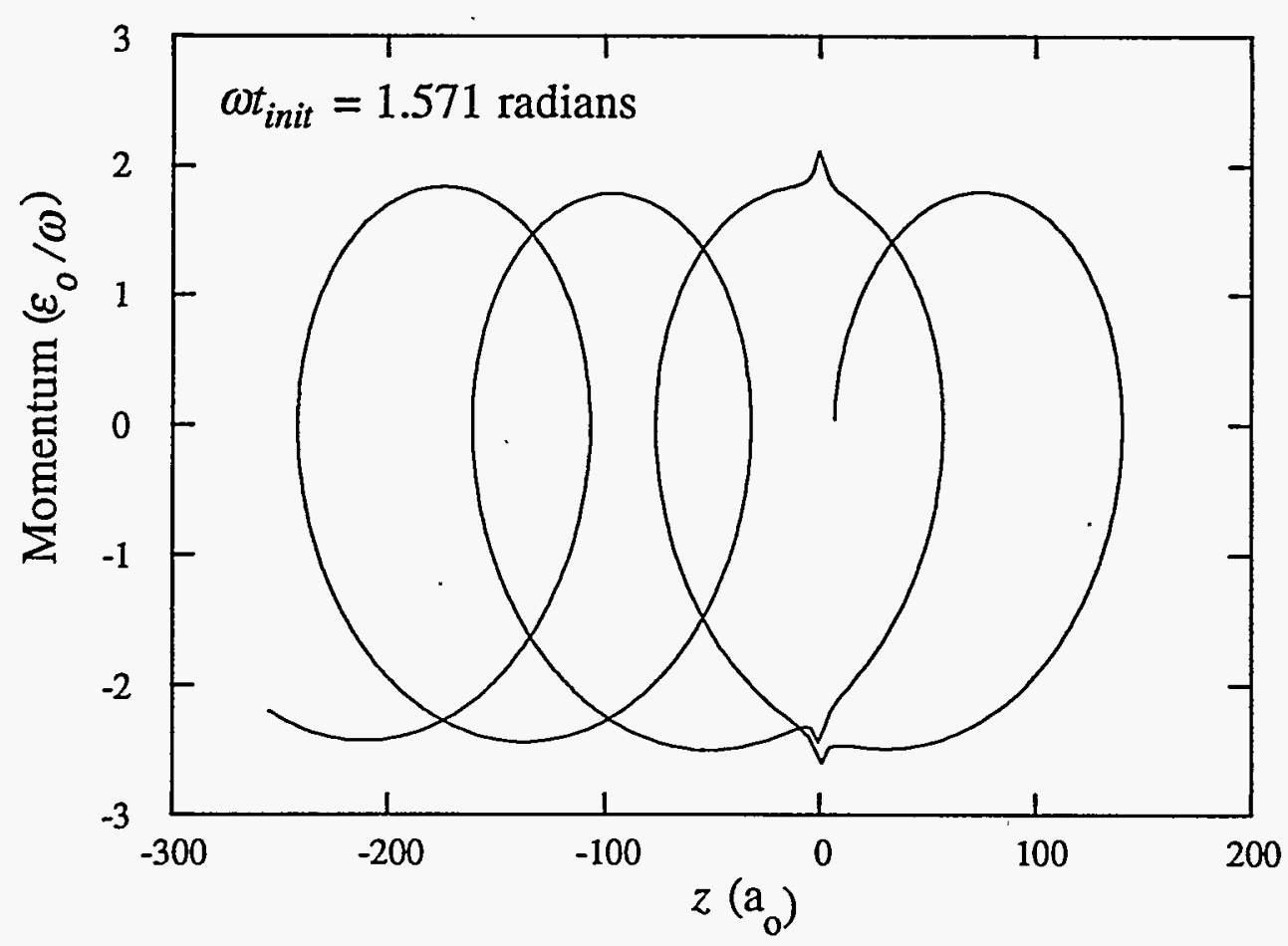

Fig. 2 Trajectory of an electron which tunneled to freedom just before the maximum in the oscillating electric field. 
back toward the core. The electron oscillates in the field as it drifts across the ion core, interacting with the core three times before finally becoming free. If the electron leaves the focal volume before the laser field turns off, the quiver energy will be converted into additional drift energy. In the short pulse case, the quiver energy is returned to the laser field and the initial drift energy will be that which is measured in the photoelectron spectrum. Without additional collisions with the nucleus the electrons would not have energies larger than $3 U_{p}, 2 U_{p}$ in short pulses, but they clearly do. It is the additional interactions with the ion core which result in the high-energy ATI peaks. Considering rescattering events with these classical electrons, we find drift energies much larger than $3 U_{p}$ can be produced.

Electrons released just after the maximum of the field during each half cycle, have the lowest initial drift velocity, are those responsible for the highest harmonic photons. When they return to the vicinity of the nucleus, they can emit a photon, being recaptured into the ground state. The energy of the emitted photon is just the return energy. By considering all possible trajectories, we find the maximum energy harmonic photon which can be produced is $\approx I_{p}+3 U_{p}$ in excellent agreement with the observed spectral cutoff. This simple quasi-classical model explains both the harmonic cutoff and the lack of one in the ATI spectrum. The reason the spectra differ is that the photoelectron energies are determined by the drift velocity at large distances from the ion core and the harmonic energies by the instantaneous velocity at the core. In fact, it is obvious that the portion of the wave packet responsible for highest energy photons does not correspond to that which ends up in the ATI peaks of the same order.

The reason that the overall strengths of the harmonic and photoelectron spectra scale similarly with intensity is that these are governed by the initiation step which is the rate at which electrons are released into the continuum. This quantum mechanical first step defines the number of electrons available to either produce photons or be measured in the ATI spectrum. It is this aspect of the ionization process which depends sensitively on the details of the atomic potential. The subsequent evolution of the electrons in the continuum should be essentially the same for all systems, depending only on the ionization potential and the ponderomotive energy.

\section{CONCLUSION}

The calculations we have presented in this paper illustrate the application of our single-active-electron model of intense-field, multiphoton processes. We have found that it is very successful in modeling very high order, non-perturbative processes in rare gas atoms. The simple quasi-classical model which accounts for many of the detailed characteristics of the photon and electron emission processes 
provides significant new insight into the excitation dynamics under intense field conditions.

\section{ACKNOWLEDGEMENTS}

The author wishes to acknowledge the substantial contributions of several colleagues to the work discussed here. The major collabor-ators have been Professors Ken Schafer (LSU) and Jeff Krause (Florida) and Dr. Louis DiMauro from Brookhaven National Laboratory. This work was carried out under the auspices of the U. S. Department of Energy at the Lawrence Livermore National Laboratory under contract number W-7405-ENG-48.

\section{REFERENCES}

1. M. D. Perry and G. Morou, Science 264, 917 (1994).

2. J. J. Macklin, J. D. Kmetic and C. L. Gordon III, Phys. Rev. Lett. 70, 766 (1993).

3. A. L'Huillier and Ph. Balcou, Phys. Rev. Lett. 70,774 (1993).

4. K. Kondo, T. Tamida, Y. Nabekawa and S. Watanabe, Phys. Rev. A 49, 3881 (1994).

5. K. C. Kulander, K. J. Schafer and J. L. Krause, Time dependent studies of multiphoton processes: In Atoms in Intense Laser Fields, Ed. M. Gavrila, Academic Press, New York (1992).

6. K. J. Schafer, The energy analysis of time-dependent numerical wave functions: In Time Dependent Methods for Quantum Dynamics, Ed. K. C. Kulander, North Holland, Amsterdam (1991).

7. J. L. Krause, K. J. Schafer and K. C. Kulander, Phys. Rev. Lett. $\underline{68}, 3535$ (1992).

8. K. J. Schafer, B. Yang, L. F. DiMauro and K. C. Kulander, Phys. Rev. Lett. 70, 1599 (1993).

9. K. C. Kulander, K. J. Schafer and J. L. Krause, Dynamics of short-pulse excitation, ionization and harmonic conversion: In Super-Intense Laser-Atom Physics, Eds. B. Piraux, A. L'Huillier and K. Rzazewski, Plenum, New York (1993).

10. P. B. Corkum Phys. Rev. Lett. 71, 1994 (1993). 


Technical Information Department $\bullet$ Lawrence Livermore National Laboratory University of California • Livermore, California 94551

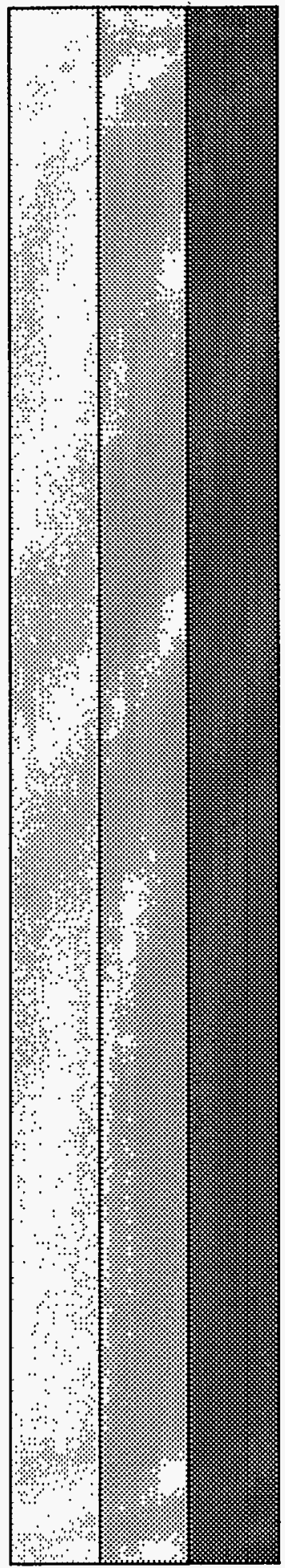

